\title{
Evaluation of the performance of irradiated silicon strip sensors for the forward detector of the ATLAS Inner Tracker Upgrade
}

R. Mori ${ }^{\mathrm{f}, *}$, P.P. Allport ${ }^{\mathrm{a}}$, M. Baca ${ }^{\mathrm{a}}$, J. Broughton ${ }^{\mathrm{a}}$, A. Chisholm ${ }^{\mathrm{a}}$, K. Nikolopoulos ${ }^{\mathrm{a}}$, S. Pyatt $^{\mathrm{a}}$,

J.P. Thomas ${ }^{\mathrm{a}}$, J.A. Wilson ${ }^{\mathrm{a}}$, J. Kierstead ${ }^{\mathrm{b}}$, P. Kuczewski ${ }^{\mathrm{b}}$, D. Lynn ${ }^{\mathrm{b}}$, M. I. Arratia-Munoz ${ }^{\mathrm{c}}$,

L.B.A. Hommels ${ }^{\mathrm{c}}$, C. Klein ${ }^{\mathrm{c}}$, M. Ullan ${ }^{\mathrm{d}}$, C. Fleta ${ }^{\mathrm{d}}$, J. Fernandez-Tejero ${ }^{\mathrm{d}}$, I. Bloch ${ }^{\mathrm{e}}$, I.M. Gregor ${ }^{\mathrm{e}}$,

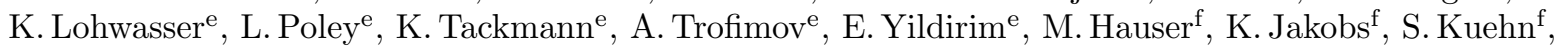
K. Mahboubi ${ }^{\mathrm{f}}$, U. Parzefall ${ }^{\mathrm{f}}$, A. Clark ${ }^{\mathrm{g}}$, D. Ferrere ${ }^{\mathrm{g}}$, S. Gonzalez Sevilla ${ }^{\mathrm{g}}$, J. Ashby ${ }^{\mathrm{h}}$, A. Blue ${ }^{\mathrm{h}}$, R. Bates ${ }^{\mathrm{h}}$, C. Buttar ${ }^{\mathrm{h}}$, F. Doherty ${ }^{\mathrm{h}}$, T. McMullen ${ }^{\mathrm{h}}$, F. McEwan ${ }^{\mathrm{h}}$, V. O'Shea ${ }^{\mathrm{h}}$, S. Kamada ${ }^{\mathrm{i}}$, K. Yamamura ${ }^{\mathrm{i}}$, Y. Ikegami ${ }^{\mathrm{j}}$,

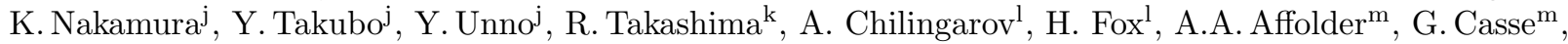

P. Dervan ${ }^{\mathrm{m}}$, D. Forshaw ${ }^{\mathrm{m}, 1}$, A. Greenall ${ }^{\mathrm{m}}$, S. Wonsak ${ }^{\mathrm{m}}$, M. Wormald $^{\mathrm{m}}$, V. Cindro ${ }^{\mathrm{n}}$, G. Kramberger ${ }^{\mathrm{n}}$,

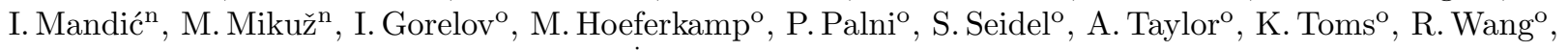
N.P. Hessey ${ }^{\mathrm{p}}$, N. Valencic ${ }^{\mathrm{p}}$, K. Hanagaki ${ }^{\mathrm{q}, \mathrm{j}}$, Z. Dolezal ${ }^{\mathrm{r}}$, P. Kodys ${ }^{\mathrm{r}}$, J.Bohm ${ }^{\mathrm{s}}$, J.Stastny ${ }^{\mathrm{s}}$, M.Mikestikova ${ }^{\mathrm{s}}$, A. Bevan ${ }^{\mathrm{t}}$, G. Beck ${ }^{\mathrm{t}}$, C. Milke ${ }^{\mathrm{u}}$, M. Domingo ${ }^{\mathrm{u}}$, V. Fadeyev ${ }^{\mathrm{u}}$, Z. Galloway ${ }^{\mathrm{u}}$, D. Hibbard-Lubow ${ }^{\mathrm{u}}$, Z. Liang $^{\mathrm{u}}$,

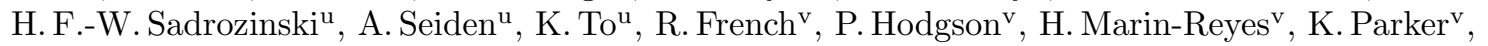

O. Jinnouchi ${ }^{\mathrm{w}}$, K. Hara ${ }^{\mathrm{x}, \mathrm{y}}$, K. Sato ${ }^{\mathrm{x}}$, K. Sato ${ }^{\mathrm{x}, \mathrm{y}}$, M. Hagihara ${ }^{\mathrm{x}}$, S. Iwabuchi ${ }^{\mathrm{x}}$, J. Bernabeu ${ }^{\mathrm{z}}$, J.V. Civera ${ }^{\mathrm{z}}$,

C. Garcia ${ }^{\mathrm{z}}$, C. Lacasta ${ }^{\mathrm{z}}$, S. Marti i Garcia ${ }^{\mathrm{z}}$, D. Rodriguez ${ }^{\mathrm{z}}$, D. Santoyo ${ }^{\mathrm{z}}$, C. Solaz ${ }^{\mathrm{z}}$, U. Soldevila ${ }^{\mathrm{z}}$

${ }^{a}$ School of Physics and Astronomy, University of Birmingham, Birmingham B15 2TT, United Kingdom

${ }^{b}$ Brookhaven National Laboratory, Physics Department and Instrumentation Division, Upton, NY 11973-5000, USA

${ }^{c}$ Cavendish Laboratory, University of Cambridge, JJ Thomson Avenue, Cambridge CB3 0HE, United Kingdom

${ }^{d}$ Centro Nacional de Microelectronica (IMB-CNM, CSIC), Campus UAB-Bellaterra, 08193 Barcelona, Spain ${ }^{e}$ DESY, Notkestrasse 85, 22607 Hambrug, Germany

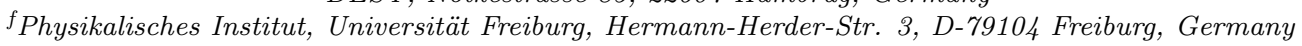

${ }^{g}$ DPNC, University of Geneva, 24, Quai Ernest-Ansermet, CH-1211 Genve 4, Switzerland

${ }^{h}$ SUPA-School of Physics and Astronomy, University of Glasgow, Glasgow G128QQ, United Kingdom

${ }^{i}$ Solid State Div., Hamamatsu Photonics K.K., 1126-1, Ichino-cho, Higashi-ku, Hamamatsu-shi, Shizuoka 435-8558, Japan ${ }^{j}$ Institute of Particle and Nuclear Study, KEK, Oho 1-1, Tsukuba, Ibaraki 305-0801, Japan

${ }^{k}$ Department of Science Education, Kyoto University of Education, Kyoto 612-8522, Japan ${ }^{l}$ Physics Department, Lancaster University, Lancaster LA14YB, United Kingdom

${ }^{m}$ Oliver Lodge Laboratory, Department of Physics, University of Liverpool, Oxford St., Liverpool L69 7ZE, United Kingdom

${ }^{n}$ Jožef Stefan Institute and Department of Physics, University of Ljubljana, Ljubljana, Slovenia

${ }^{\circ}$ Department of Physics and Astronomy, University of New Mexico, MSC07 4220, 1919 Lomas Blvd. NE, Albuquerque, NM 87131, USA

${ }^{p}$ Nikhef, Science Park 105, 1098 XG Amsterdam, Netherlands

${ }^{q}$ Department of Physics, Osaka University, Machikaneyama-cho 1-1, Toyonaka-shi, Osaka 560-0043, Japan

${ }^{r}$ Charles University in Prague, Faculty of Mathematics and Physics, V Holesovickach 2, Prague 8, Czech Republic

${ }^{s}$ Academy of Sciences of the Czech Republic, Institute of Physics, Na Slovance 2, 18221 Prague 8, Czech Republic

${ }^{t}$ School of Physics and Astronomy, Queen Mary University of London, London E1 4NS, United Kingdom

${ }^{u}$ Santa Cruz Institute for Particle Physics (SCIPP), University of California, Santa Cruz, CA 95064, USA

${ }^{v}$ Department of Physics and Astronomy, The University of Sheffield, Hicks Building, Hounsfield Road, S3 7RH Sheffield, United Kingdom

${ }^{w}$ Institute of Science and Engineering, Tokyo Institute of Technology, Ookayama 2-12-1, Meguro-ku, Tokyo 152-8551, Japan

${ }^{x}$ Institute of Pure and Applied Sciences, University of Tsukuba, Tsukuba, Ibaraki 305-8751, Japan

${ }^{y}$ Center for Integrated Research in Fundamental Science and Engineering, University of Tsukuba, Tsukuba, Ibaraki 305-8571 Japan

${ }^{z}$ IFIC/CSIC-UVEG, Ed. Inst. Investigacion, PO Box 22085, 46071 Valencia, Spain

\begin{abstract}
The upgrade to the High-Luminosity LHC foreseen in about ten years represents a great challenge for the ATLAS inner tracker and the silicon strip sensors in the forward region. Several strip sensor designs were developed by the ATLAS collaboration and fabricated by Hamamatsu in order to maintain enough performance in terms of charge collection efficiency and its uniformity throughout the active region. Of
\end{abstract}


particular attention, in the case of a stereo-strip sensor, is the area near the sensor edge where shorter strips were ganged to the complete ones. In this work the electrical and charge collection test results on irradiated miniature sensors with forward geometry are presented. Results from charge collection efficiency measurements show that at the maximum expected fluence, the collected charge is roughly halved with respect to the one obtained prior to irradiation. Laser measurements show a good signal uniformity over the sensor. Ganged strips have a similar efficiency as standard strips.

Keywords: irradiated silicon strip sensor, charge collection, ATLAS Inner Tracker, HL-LHC.

\section{Introduction}

The High-Luminosity Large Hadron Collider (HL-LHC) [1, starting with the Phase-II upgrade, is foreseen in about ten years. The beam luminosity will reach more than $5 \times 10^{34} \mathrm{~cm}^{-2} \mathrm{~s}^{-1}$ demanding a major upgrade of the detectors in various experiments. According to the Letter Of Intent 2, the Inner Tracker (ITk) [3] of the ATLAS Experiment will be based on silicon pixel and strip modules, the latter disposed in six barrel layer and seven discs constituting the endcap.

The development of strip sensors in the endcap region represents a great challenge for several reasons. Firstly, in the forward region a maximum fluence of $1.6 \times 10^{15} n_{e q} \mathrm{~cm}^{-2}$ (with a safety factor of 2) is foreseen 2. Secondly, the disc geometry and the aim to reduce the area of dead regions with the strip layout require sensors containing strips with different length. Indeed, in order to maintain high resolution (small pitch) and tracking performance (stereo angle between front and back side planes) the sectors of the disc have to be covered by strips not parallel to the radial direction. Covering the full area, the strips will have shorter length going toward the sector side. In particular, the disc sectors (Petals) will be divided in rings with trapezoidal-like sensor shapes. Radial strips following the planned stereo angle are not parallel to the trapezoidal sides and the more external ones will have shorter lengths because reaching the sensor lateral side. Two orphan strips at two corners of the sensor cannot be connected directly to the readout electronics.

The first challenge, i.e. the radiation hardness, has been dealt within collaborations like RD50 44 and resulted in major changes with respect to the current Semiconductor Tracker (SCT) [5]. Most of all, the new tracker will embed p-type sensors instead of n-type, the former not suffering of type inversion 6 . Another aspect related to the harsh environment is the stability during long time operations. Recently it was observed that the performance of irradiated sensors decreases when they operate at high voltage for long time [7. Notably, this occurs for very high voltages $(>1000 \mathrm{~V})$, but a quality control in term of long term stress is mandatory.

With respect to the second challenge of the dead regions, Hamamatsu proposed to gang orphan strips to complete ones (single) maintaining the capability to discriminate between charge collection events in the two regions.

This work is focused on the investigation of performance of the endcap ATLAS12A sensors [8, 9 , produced by Hamamatsu. Charge collection as a function of bias voltage characteristics of sensors irradiated with $\gamma$-rays and protons at different doses and fluences, as well as the stability in time, are presented. They are evaluated in comparison with the performance of ATLAS12 barrel sensors and the previous ATLAS07 layout sensors. Results concerning the relative efficiency of the orphan strips with respect to the complete strips are shown.

\footnotetext{
*Corresponding author. e-mail: riccardo.mori@physik.uni-freiburg.de
}

${ }^{1}$ Now at Syracuse University. 


\section{Materials}

\subsection{Samples}

ATLAS12 sensors are $\mathrm{n}^{+}$-on-p strip sensors produced on Float Zone substrate, $6^{\prime \prime}$ diameter, $310 \pm 25 \mu \mathrm{m}$ thick, $<100>$ oriented wafers $[10$. The resistivity is around $3.5 k \Omega$, resulting in a depletion voltage of about $350 \mathrm{~V}$.

Sensors have $16 \mu \mathrm{m}$ implant and different strip pitches for barrel and endcap geometries. In this work, tests have been performed in miniature endcap sensors with the ATLAS12A layout. They are of $1 \times 1 \mathrm{~cm}^{2}$ area, with pitches from 63.6 to $65.1 \mu \mathrm{m}$ (small pitch) and from 102 to $105 \mu \mathrm{m}$ (large pitch), embedding p-stops of $6 \mu \mathrm{m}$ width. The miniatures reproduce the tilted strips of the endcap layout, having the most external strips which ends on the sensor lateral side (close the bias ring) and not to the bottom like the internal ones. AC and DC ganging of these orphan strips was implemented as in Fig. 1] 9. The trick for maintaining resolution
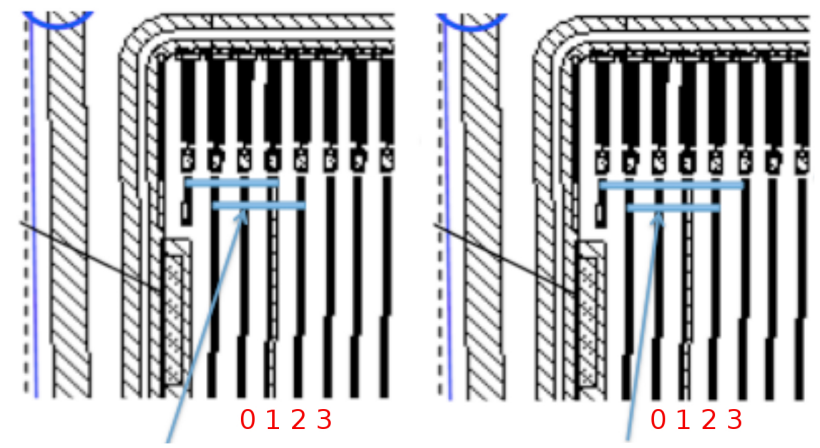

Figure 1: Ganging layout. DC ganging (left) and AC ganging (right) implemented on the miniature sensor under test 9]. The two left most strips, orphans, are connected to neighbours as indicated by the arrows and the horizontal segments; DC ganging connect the orphan metal to the neighbour implant, while AC ganging connect the orphan metal to the neighbour metal.

in the critical region is to connect the orphan strips not to the first, but to the farther neighbour strips so that the readout of a sparse cluster from the ganged and the most external single strip (e.g. channels 2 and 0 in Fig. 1 (right), AC ganging) will identify an event close to the orphan, while a single cluster readout from the ganged and the first neighbour (e.g. channels 2 and 1) will identify an event close to the ganged long strip. It should be noticed that in the case of DC ganging, a hit read by the 2 ganged strips (channels 1 and 2) has a redundancy because it can correspond to a particle between the two orphan or between the two long strips. The hit position would be resolved considering the information from other planes.

In order to discriminate surface and bulk damage, sensors were irradiated with $\gamma$-rays at $1,3,10 \mathrm{Mrad}$ doses and with $23 \mathrm{MeV}$ protons at $5 \times 10^{14}, 1 \times 10^{15}, 2 \times 10^{15} n_{e q} \mathrm{~cm}^{-2}$. All the sensors were also tested after annealing of 80 minutes at $60^{\circ} \mathrm{C}$. Laser measurements were performed only in annealed samples having small pitch.

\subsection{Experimental set-up}

Tests aimed to measure the absolute charge collection were performed with a ${ }^{90} \mathrm{Sr}$ source giving MIP-like beta particles. Triggering for the readout is given by two scintillators in coincidence, which are also used to discard lower energetic particles. Sensors under test were placed in a freezer while temperature and humidity were constantly monitored.

Tests of the orphan strip performance, requiring high spatial resolution, were performed with a laser of $971 \mu \mathrm{m}$ wavelength with about $100 \mu \mathrm{m}$ penetration depth and with a FHWM down to $4 \mu \mathrm{m}$ in the focus. In this case, sensors under test were placed in a motorized $\mathrm{x}-\mathrm{y}$ stage with about $0.5 \mu \mathrm{m}$ accuracy. Cooling is performed with liquid nitrogen vapours down to less than $-15^{\circ} \mathrm{C}$.

In both the beta-source and the laser set-up, sensor biasing and current readout was performed with a Keithley 237. Signal readout was performed using the ALiBaVa system [11, with the Beetle chip configured to have a peaking time of about $22 \mathrm{~ns}$. 


\section{Signal analysis}

All the beta-source measurements on irradiated sensors shown here were performed at about $-16^{\circ} C$ temperature and $4 \%$ relative humidity, and non-irradiated sensors at room temperature. Measurements were taken up to $1000 \mathrm{~V}$ bias voltage. Only for a few sensors it was not possible to go up to $1000 \mathrm{~V}$ due to the fact that the breakdown point was reached before.

The analysis is performed with a custom software. After pedestal and common mode subtraction, a residual cut selecting only strips in the trajectory from the source and the scintillator area was applied. Events occurring within the $10 \mathrm{~ns}$ interval around the signal peak were selected in order to obtain high statistic and relatively small underestimation (less than 5\%). Clustering with cuts on the seed channel of 3.5 and on the neighbour channels of 1.8 times the noise values was performed in order to exclude fake hits and noise contributions to the signal.

Laser measurements on irradiated sensors are performed at temperatures lower than $-15^{\circ} \mathrm{C}$, but due to the length of the measurement (up to one day) and the unstable nitrogen flow, changes of several degrees could occur. Test measurements with the same set-up and similar sensor showed that a difference of about $10^{\circ} \mathrm{C}$ has a negligible effect on the charge collection. Large areas (up to $500 \times 250 \mu \mathrm{m}^{2}$ ) around the shorter orphan strip were scanned in steps of $2 \mu \mathrm{m}$, scanning lines in the direction perpendicular to the strips $(250 \mu \mathrm{m})$ and collecting 500 pulses per point. Measurements shown here were performed at $500 \mathrm{~V}$. The signal at the pulse peaking time was recorded.

After pedestal and common mode subtraction, two dimensional maps (e.g. Fig. 5) were aligned in both plane directions for observation of the overall results. Profile lines perpendicular to the strips and in the region of interest were analysed and normalized with respect to the maximum charge collection of the single strip.

\section{Results}

\subsection{Beta-source measurements}

In Fig. 2 charge collection measurements as a function of bias voltage of $\gamma$-irradiated samples are shown together with a non-irradiated sample (as-grown). For every dose, and even after annealing, there is no

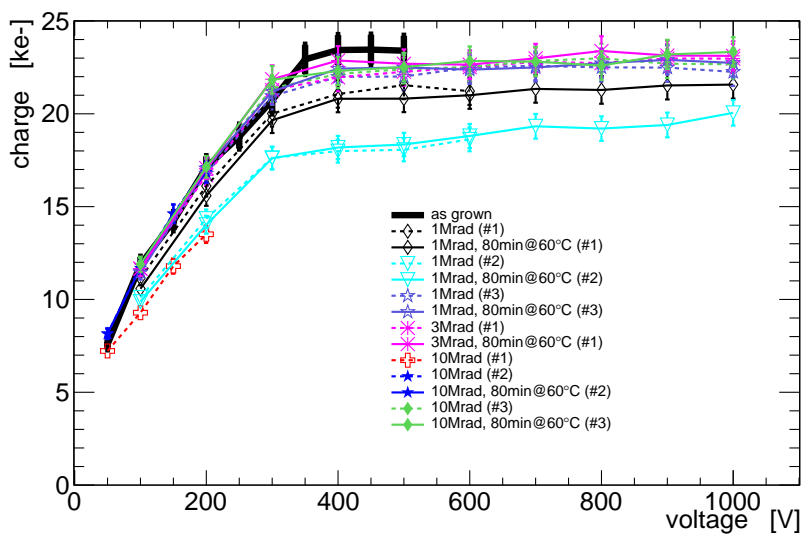

Figure 2: Charge collection versus voltage of $\gamma$-irradiated samples. More sensors per fluence were tested (sensor index in brakets).

evidence of a change in collected charge. Only one sensor showed lower charge probably due to a slightly worst production, but it still displayed saturation of charge collection at a similar voltage.

The charge collection of proton-irradiated samples, before and after annealing, is shown in Fig. 3 . Sensors irradiated with the same fluence presented similar performance with a difference less than $1.5 \mathrm{ke}^{-}$in charge collection. The charge collection drops significantly for higher fluences, down to $7 k e^{-}$at $500 \mathrm{~V}$ for the 

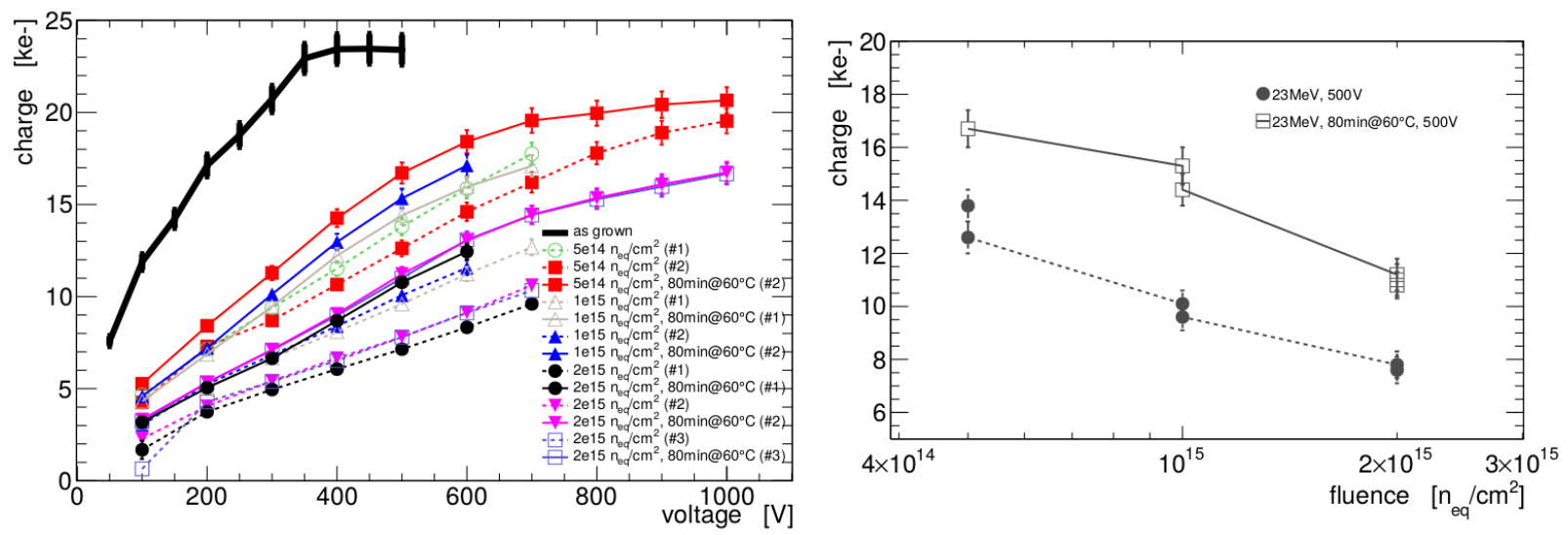

Figure 3: Charge collection vs. bias voltage (left) and vs. fluence (right) of proton-irradiated samples. More sensors per fluence were tested (sensor index in brakets).

$2 \times 10^{15} n_{e q} \mathrm{~cm}^{-2}$ irradiated samples. Annealing for 80 minutes at $60^{\circ} \mathrm{C}$ give a significant recovery (e.g. about $2 \mathrm{ke}^{-}$at $500 \mathrm{~V}$ for the $2 \times 10^{15} \mathrm{n}_{e q} \mathrm{~cm}^{-2}$ irradiated samples). Moreover, beneficial annealing is more effective for lower bias voltages (below $500 \mathrm{~V}$ ). As a significant value for the LHC operations, charge collection at $500 \mathrm{~V}$ of the $2 \times 10^{15} n_{e q} \mathrm{~cm}^{-2}$ irradiated and annealed samples is almost halved, but still very much above the expected noise level (less than $1 k e^{-}$for irradiated samples).

This result is in agreement with results from barrel sensors [12] and ATLAS07 sensors 13 which showed $11 \mathrm{ke}^{-}$at $500 \mathrm{~V}$ for fluences in between 1.7 and $2.5 \times 10^{15} n_{e q} \mathrm{~cm}^{-2}$.

The charge collection of a sensor tested at $600 \mathrm{~V}$ and at $1100 \mathrm{~V}$ for about three days shows no drop. Figure 4 shows the result of the test at $1100 \mathrm{~V}$. This is in agreement with measurements of a constant

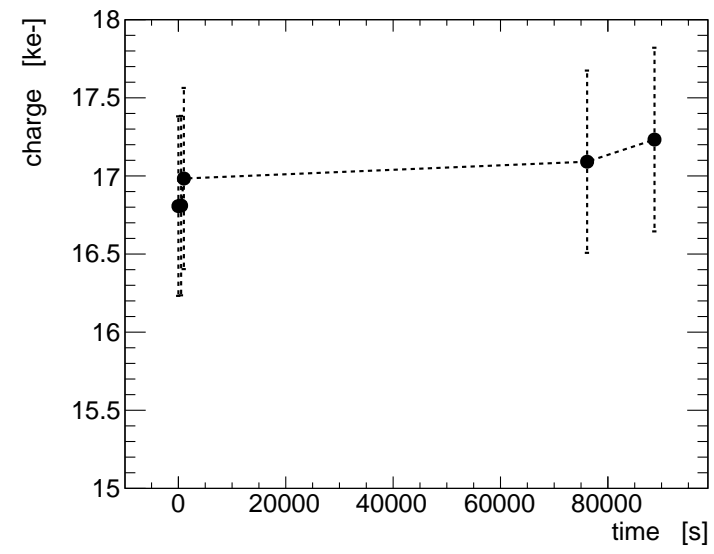

Figure 4: Stability performance. Charge collection vs. time at $1100 \mathrm{~V}$ of a sample irradiated with protons at $2 \times 10^{15} n_{e q} \mathrm{~cm}^{-2}$.

interstrip capacitance in similar sensors 14. It should be noted that ATLAS07 samples irradiated at the same fluence and tested at the same voltage showed a drop of about $3 k e^{-}$after 3 days [15, and similar ATLAS07 sensors a change in the interstrip capacitance.

\subsection{Laser measurements}

As an example, Fig. 5 shows the two-dimensional collection map from a laser measurement in a sensor irradiated at $2 \times 10^{15} n_{e q} \mathrm{~cm}^{-2}$ and annealed, having small pitch and AC ganging. Strips are oriented parallel to the $x$ direction. It can be noticed that when the laser is above the aluminium metallisation of 
the strip, light is reflected and negligible charge is collected. In Fig. 5 low signal lines at $y \sim 100 \mu m$ and $y \sim 420 \mu \mathrm{m}$ (left), and $y \sim 240 \mu \mathrm{m}$ (right) are shown. Areas with higher charge collection are also visible in correspondence of the DC pads (e.g. three spots on the left plot, $x>140 \mu \mathrm{m}$ ). The typical relative charge
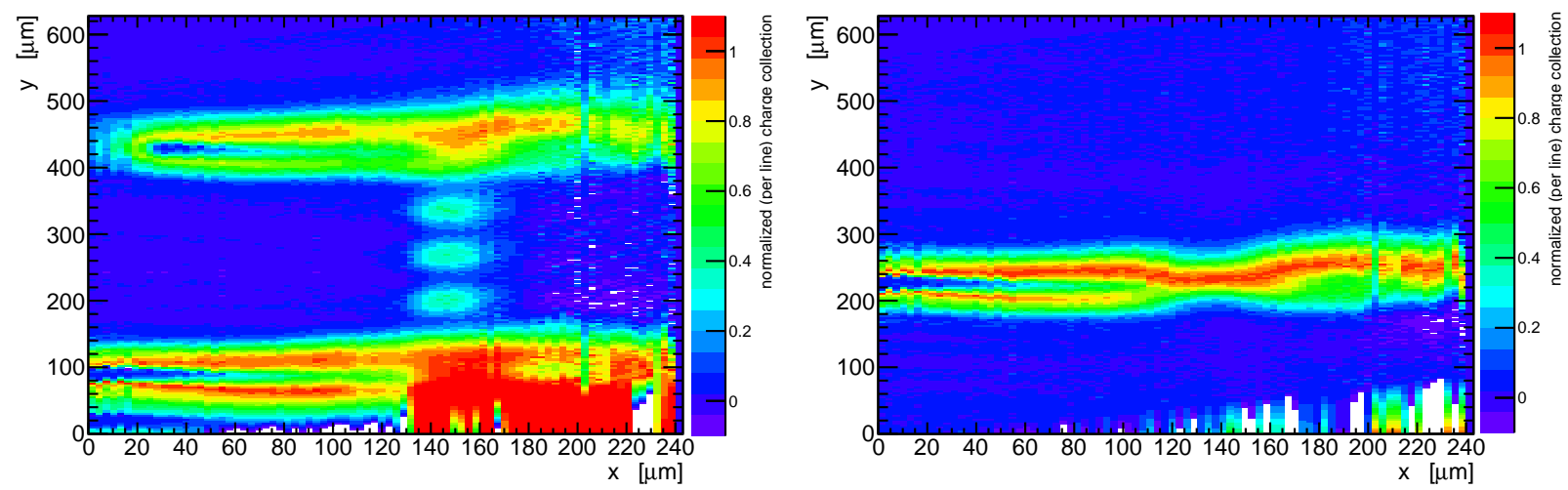

Figure 5: Laser measurements. Normalized charge collection maps of a ganged strip channel (left) and a single strip channel (right) of a sample irradiated with protons at $2 \times 10^{15} n_{e q} \mathrm{~cm}^{-2}$, AC ganged.

collection profile of a single strips has two peaks and decreases with the distance from the strip center; charge collection is almost zero in correspondence of the aluminium. Results from non-irradiated and irradiated with $\gamma$-rays samples, having small pitch and DC ganging, are shown in Fig. 6. Both AC and DC ganged
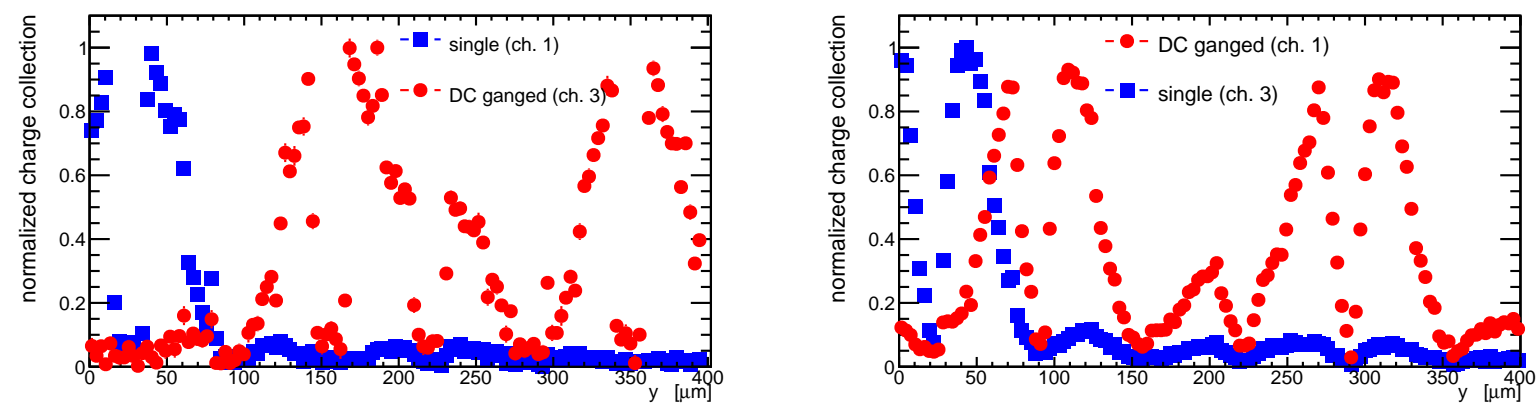

Figure 6: Performance of non-irradiated sample (left) and a sample irradiated at 1 Mrad $\gamma$-rays dose (right), DC ganged. Normalized charge collection profile.

non irradiated samples show that the charge collection of the ganged strip is similar to the one of the single strips, when the laser is close to the orphan strip or to the long strip $(y \sim 150 \mu m$ and $y \sim 350 \mu m)$. It should be considered that the ganged strip has significant charge collection when the laser is on the far side of the neighbour strip $(y \sim 250 \mu \mathrm{m})$. The sample irradiated with $\gamma$-rays shows a relevant drop in the charge collection of the ganged strip.

Figure 7 shows the charge collection profiles of samples irradiated with $1 \times 10^{15}$ and $2 \times 10^{15} n_{e q} \mathrm{~cm}^{-2}$ proton fluences, having DC ganging. The ganged strip presented a drop of charge collection especially at $2 \times 10^{15} n_{e q} \mathrm{~cm}^{-2}$, but it was still able to collect about $90 \%$ of the charge collected by the single strips.

In Fig. 8 the relative charge collection of a sample irradiated at $2 \times 10^{15} n_{e q} \mathrm{~cm}^{-2}$ with AC ganging is shown. The charge collection of the ganged strip is close to the one of the single strip, with only a drop of about $15 \%$ in the orphan strip region. In this case, the charge collection when the laser hit the neighbour region is lower than for the DC ganged sensor.

Measurements on a sensor irradiated at $1 \times 10^{15} n_{e q} \mathrm{~cm}^{-2}$, DC ganged, and tested at 50 and $500 \mathrm{~V}$, show no significant dependence on the bias voltage. The relative charge collection of the ganged strip is always above $90 \%$. 

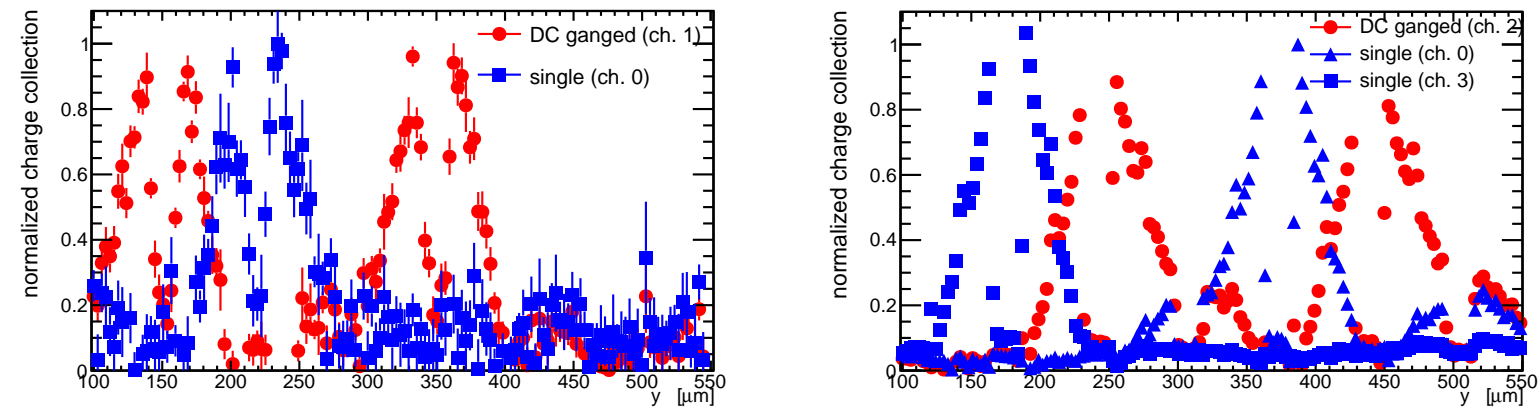

Figure 7: Performance of samples irradiated with protons at $1 \times 10^{15}$ (left) and $2 \times 10^{15} n_{e q} \mathrm{~cm}^{-2}$ (right), with small pitch, DC ganged. Normalized charge collection profile.

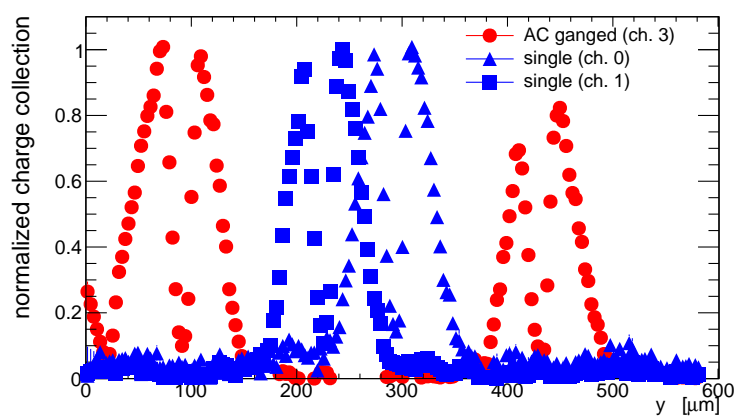

Figure 8: Performance of a sample irradiated with protons at $2 \times 10^{15} n_{e q} \mathrm{~cm}^{-2}$, with small pitch, AC ganged. Normalized charge collection profile.

\section{Discussion}

Charge collection results show a degradation of the performance with proton fluence in agreement with barrel and ATLAS07 sensors. It is noticeable that they show the same radiation hardness although they have significantly lower resistivity with respect to the ATLAS07. Of particular interest is also the stability in time of the charge collection, correlated to the stability of the interstrip capacitance. A systematic study with more sensors needs to be carried out, but the difference to the ATLAS07 performance is remarkable as many ATLAS07 sensors showed a significant drop in performance.

Laser measurements were presented without giving numerical results. This is because those measurements are influenced by many factors, first of all the precision of the alignment. The duration of a measurement cannot ensure stability in this respect, therefore single profiles in the region of interest were preferred to large area profiles. Despite of this, comparing the relative charge collection of ganged strips to single ones at the same line scan allows us to draw essential conclusions. Measurements on more than one single strip for the same line (e.g. in Fig. 8) show the same amount of charge collection which allows to exclude distortion of the efficiency due to laser defocusing in the profile direction.

It is interesting to observe the charge collection of strips when the laser is close to the next neighbour (e.g. collection of ganged strip at $y \sim 340 \mu \mathrm{m}$ in Fig. 7). This is partially resulting from the charge cloud dispersion, but another significant contribution occurs due to the cross-talk of channels, eventually amplified by the ganging connection which is almost crossing the other neighbour strips. Indeed, not negligible amount of charge collection was collected with the laser, also on the second far neighbour. Cross-talk seems to be more for DC ganged samples (comparing Fig.7 with Fig.8) and increasing towards the ganging connection (see Fig 9 at $x>200 \mu m$ ), but this needs to be concluded after a more systematic study on more samples. In any case, it is negligible for the operations. Finally, it should be mentioned that a slightly higher reduction in the charge collection of the ganged strip has been observed in the proximity of the ganging connection. 


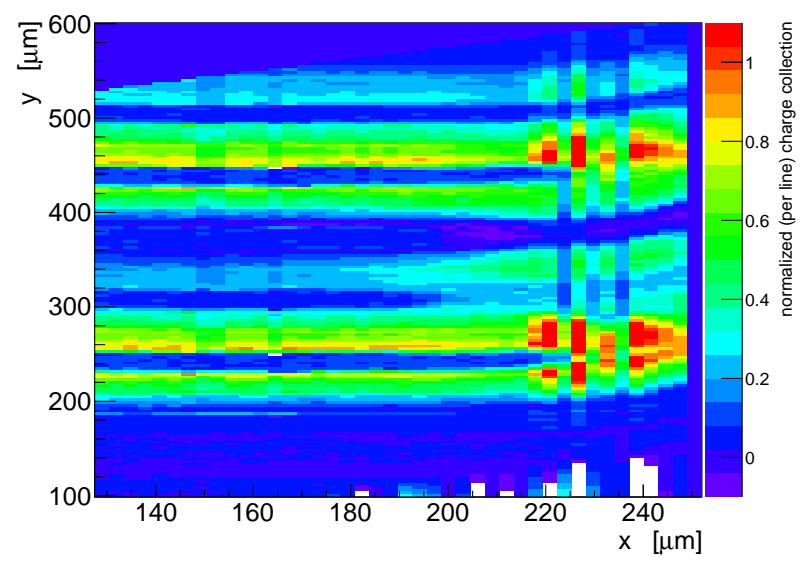

Figure 9: Performance of a sample irradiated with protons at $2 \times 10^{15} n_{e q} \mathrm{~cm}^{-2}$, DC ganged. Normalized charge collection map of a ganged strip channel.

\section{Conclusion}

Many ATLAS12A sensors with endcap geometry, irradiated with $\gamma$-rays and protons, were systematically tested by measuring the charge collection performance. The absolute value of the charge collection was obtained with beta-source measurements, while performance of critical regions was tested with laser measurements.

Beta-source measurements show that the charge collection for these sensors at a bias voltage of $500 \mathrm{~V}$ halves at fluences of about $1 \times 10^{15} n_{e q} \mathrm{~cm}^{-2}$ or $2 \times 10^{15} n_{e q} \mathrm{~cm}^{-2}$ annealed for 80 minutes at $60{ }^{\circ} \mathrm{C}$, similar to the previous ATLAS07 layout sensors. No long term performance decrease was observed (in contrast to the ATLAS07 sensors). The ganging of the orphan strips allows maintaining significant tracking performance also in the critical regions not covered by readout strips. The amount of collected charge of orphan strips is almost at the level of single ones, the worst case is $10 \%$ loss for the $2 \times 10^{15} n_{e q} \mathrm{~cm}^{-2}$ irradiated samples. A small cross-talk due to the ganging connection was observed, especially for DC ganged sensors.

\section{Acknowledgements}

The irradiations were performed: with protons at the University of Birmingham MC40 cyclotron, supported by the H2020 project AIDA-2020, GA number 654168, and the UK's Science and Technology Facilities Council, at Cyclotron and Radioisotope Center (CYRIC), Tohoku University, with Y. Sakemi, M. Ito, and T. Wakui, at the Karlsruhe Institute of Technology (KIT) by A. Dierlamm, supported by the Initiative and Networking Fund of the Helmholtz Association, contract HA-101 (Physics at the Terascale) and the European Commission under the FP7 Research Infrastructures project AIDA, Grant agreement no.262025, and at the LANSCE facility, Los Alamos National Laboratory; with neutrons at JSI TRIGA reactor in Ljubljana supported by the H2020 project AIDA-2020, GA no. 654168; with pions at Paul Scherrer Institut (PSI); and with $\gamma$ 's at Brookhaven National Laboratory (BNL).

The research was supported and financed in part by the Ministry of Education, Youth and Sports of the Czech Republic (Grant No. LG13009), the German Federal Ministry of Education and Research, and the Helmholtz Association, the European Social Fund and by the Ministry of Science, Research and Arts, Baden-Württemberg, Germany, the Japan Society for Promoting Science KAKENHI-A Grant number 20244038 and KAKENHI-C Grant number 20540291, the Ministry of Education, Culture, Sports, Science and Technology-Japan, KAKENHI for Research on Priority Area Grant number 20025007 and for Scientific Research on Innovative Areas Grant number 23104002, the Slovenian Research Agency, the Spanish Ministry of Economy and Competitiveness through the Particle Physics National Program (ref. FPA2012-39055C02-01 and FPA2012-39055-C02-02) and co-financed with FEDER funds, the financial support of the State 
Secretariat for Education, Research, and Innovation, the Swiss National Science Foundation and the Canton of Geneva, Switzerland, the UK Science and Technology Facilities Council (under Grant ST/M006409/1), and the United States Department of Energy, grant DE-FG02-13ER41983.

\section{References}

[1] S. McMahon, these proceedings, 2015

[2] ATLAS Collaboration, Tech. Rep. CERN-LHCC-2012-022. LHCC-I-023 (2012)

[3] I.-M. Gregor, these proceedings, 2015.

[4] M. Moll, RD50 Status Report 2009/2010 - Radiation hard semiconductor devices for very high luminosity colliders, Tech. Rep. CERN-LHCC-2012-010. LHCC-SR-004, CERN, Geneva (Jun 2012).

[5] A. Ahmad, et al., The silicon microstrip sensors of the atlas semiconductor tracker, Nucl. Instr. and Meth. A 578 (1) (2007) $98-118$.

[6] G. Casse, P. Allport, M. Hanlon, Nuclear Science, IEEE Transactions on 47 (3) (2000) 527-532.

[7] R. Mori, et al., Nucl. Instr. and Meth. A 796 (2015) $131-135$.

[8] Y. Unno, et al., Nucl. Instr. and Meth. A 765 (2014) 80-90.

[9] ATLAS Collaboration, Supply of silicon microstrip sensors of ATLAS12 specification, Tech. rep. (2014).

[10] G. Calefato, et al., A comparison on radiation tolerance of 100 and 111 silicon substrates of microstrip detectors, Nucl. Instr. and Meth. A 476 (3) (2002) $744-750$.

[11] R. M. Hernández, Nucl. Instr. and Meth. A 623 (2001) 207-209.

[12] K. Hara, these proceedings, 2015.

[13] Y. Unno, et al., Nucl. Instr. and Meth. A 636 (1, Supplement) (2011) S24 - S30.

[14] M. Mikestikova, these proceedings, 2015.

[15] R. Mori, et al., Longterm performance of silicon strip detectors under high bias voltage: charge collection and laser measurements, presented at RD50 Workshop, Santander, Spain, 22 June 2015. 